\title{
A class of joint production and transportation planning problems under different delivery policies
}

\author{
Utku Koc, Ayşegül Toptal *, Ihsan Sabuncuoglu \\ Industrial Engineering Department, Bilkent University, Ankara, 06800, Turkey
}

\section{A R T I C L E I N F O}

\section{Article history:}

Received 1 May 2012

Received in revised form

5 November 2012

Accepted 5 November 2012

Available online 17 November 2012

\section{Keywords:}

Supply chain scheduling

Outbound transportation

Cargo capacity

Production/transportation

\begin{abstract}
A B S T R A C T
This paper examines a manufacturer's integrated planning problem for the production and the delivery of a set of orders. The manufacturer in this setting can use two vehicle types for outbound shipments. The first type of vehicle is available in unlimited numbers, but expensive. The second type, which is relatively low in its price, has limited and time-varying availability. We analyze the manufacturer's planning problem under different delivery policies characterized by each of the following: whether orders can be split or not, whether they can be consolidated or not, and whether their sizes are restricted to be in integer multiples of vehicle capacities or not.
\end{abstract}

(c) 2012 Elsevier B.V. All rights reserved.

\section{Introduction and related literature}

We study a manufacturer's multi-period planning problem to produce and ship a certain number of orders before their deadlines with the minimum inventory holding and transportation costs. The manufacturer can use two types of vehicles for outbound deliveries. The two vehicle types differ in their availability and costs. The first type of vehicle is available in unlimited numbers in all periods, however, it is more costly. The second type of vehicle, which is more economical, has time-varying and limited availability. We study the manufacturer's planning problem in this setting under the following different delivery characteristics:

- Orders allowed to be consolidated (Consolidate) or not (NoConsolidate). If consolidation of orders is allowed, then different orders can be bundled and shipped together in the same vehicle. Consolidation may reduce the number of vehicles used, and thereby transportation costs, particularly when order sizes are small and/or customers are in close geographical proximity. However, for many practical reasons, consolidation may be ruled out at the planning phase (i.e., "No-Consolidate"). Such reasons include special handling needs, geographic constraints, laws or trade agreements in cross-border transactions or having direct competitors as customers who do not collaborate.

- Orders allowed to be split (Split) or not (No-Split). Splitting refers to delivering the portions of an order at multiple points in time. Allowing for orders to be split may reduce inventory

\footnotetext{
* Corresponding author.

E-mail address: toptal@bilkent.edu.tr (A. Toptal).
}

holding costs, improve service levels, or mitigate the risks of loss or damage during loading and unloading. However, as Chen and Pundoor [3] report, for ease in tracking and handling, customers may want their orders to be delivered as a whole rather than split (i.e., "No-Split").

- Size of orders in terms of vehicle/container capacities. We consider two cases depending on the restrictions imposed by suppliers on order sizes. In some applications, suppliers accept order sizes only in integer multiples of vehicle/container capacities and dispatch in full truck loads (FTL). This practice may enable more economical shipments and sturdy loading, which helps to prevent breakage. We refer to the problem settings with this restriction as having FTL-Delivery characteristic. We use the term General-Delivery as a characteristic to identify the settings with no such restriction on order and dispatch sizes.

Considering all possible combinations of the different delivery characteristics, we identify six policies for outbound deliveries. Those are; Consolidate and Split, No-Consolidate and Split, Consolidate and No-Split, No-Consolidate and No-Split, FTL-Delivery with Split, FTL-Delivery with No-Split. Note that, consolidating multiple orders in the same truck is not relevant in the case of FTL-Delivery, as the demand sizes and the delivery sizes of all orders are integer multiples of the vehicle capacity. We consider the question of how the manufacturer plans for production and transportation under each policy as a different problem. These problems, indexed from one to six, are summarized in Table 1. For example, Problem 1 refers to the planning problem under a Consolidate and Split Policy.

Several studies have been conducted on integrated production and outbound planning problems (e.g., Li and Ou [4], Chen and 
Table 1

A summary of the problems under different delivery policies.

\begin{tabular}{llll}
\hline & General-Delivery & & FTL-Delivery \\
\cline { 2 - 3 } & Consolidate & No-Consolidate & \\
\hline Split & Problem 1 & Problem 2 & Problem 5 \\
No-Split & Problem 3 & Problem 4 & Problem 6 \\
\hline
\end{tabular}

Lee [2] and Zhong et al. [8]). We cite Chen [1] for a review of the literature covering this area. It is important to note that a majority of the studies on integrated production and outbound planning assume that there is only one type of vehicle available. Wang and Lee [7], Stecke and Zhao [6], and Chen and Lee [2] are examples of the few studies that model different types of vehicles. In all these papers, vehicles are considered as heterogeneous due to the differences in their speed and cost. Mainly, it is assumed that the speedier vehicle type is more costly. Our first contribution to the literature is that we model the existence of heterogeneous vehicles that are different in their availability and costs. This may occur in practice for many reasons, e.g., the presence of multiple third-party logistics (3PL) providers, or pricing strategy of a 3PL company. Our second contribution is that we introduce a new class of problems based on different delivery policies for the setting of interest. We establish their complexity statuses by either providing a pseudopolynomial algorithm or proving that no such algorithm exists.

In the next section, we provide more details about the problem setting and a generic mathematical formulation. In Section 3, we discuss some optimality properties that are common under all delivery policies. The analysis for the problems under the General-Delivery characteristic, those are the problems numbered 1 through 4, is presented in Section 4 . A similar discussion follows in Section 5 for the problems under the FTL-Delivery characteristic. We conclude the paper in Section 6 with a summary of the findings.

\section{Problem definition and formulation}

The different delivery characteristics considered for the manufacturer lead to six problems. In these six problems, the manufacturer has to decide the production and delivery schedules of $n$ orders over a finite horizon of $T$ periods. The production capacity in period $t$ is limited by $P_{t}$ units. The demand for order $i$, that is $S_{i}$ units, has to be satisfied promptly before deadline $D_{i}$. A holding cost of $H_{t}(I)$ is incurred for carrying $I$ units of inventory from period $t$ to $t+1$. Orders can be shipped to the customers using two types of vehicles, those are Types I and II. All vehicles are identical in their capacity (i.e., size capacity of $K$ units). The objective of the manufacturer is to minimize the sum of inventory holding costs and transportation costs without any job being tardy. Order acceptance and rejection decisions are made in advance and a feasible schedule exists for any instance of the six problems.

The manufacturer incurs the costs of delivery to the customers, all of whom are located in close proximity to one another. A combination of Types I and II vehicles can be used by the manufacturer. Type I vehicles are available in unlimited numbers in all periods, whereas a limited number, $A_{t}$, of Type II vehicles are available in period $t$. It costs $C_{1, t}(x)$ money units to utilize $x$ number of Type I vehicles in period $t$, including the operating costs (e.g., fuel expenditure, driver wages, etc.), and environmental costs (e.g., emission cost, waste disposal cost, etc.). Similarly, the cost of utilizing $x$ number of Type II vehicles in period $t$ amounts to $C_{2, t}(x)$ money units. Type II vehicles - when they become available - can be held at the facility, to be used in future periods. In this case, a waiting cost of $W_{t}(w)$ is incurred for carrying $w$ vehicles from period $t$ to $t+1$. The cost terms introduced above satisfy the following conditions at all periods $t$ :

- $C_{1, t}(0)=0$ and $C_{2, t}(0)=0$.
- $C_{1, t}(x)>C_{2, t}(x)$ for $x>0$.

- $C_{1, t}(x+1)-C_{1, t}(x)>C_{2, t}(y+1)-C_{2, t}(y)>0$ where $x \geq 0$ and $y \geq 0$.

- $H_{t}(I+1)>H_{t}(I)$ for $I \geq 0$.

- $W_{t}(w+1)>W_{t}(w)$ for $w \geq 0$.

The first condition simply implies that the transportation cost due to any vehicle type is zero if no vehicles of that type are used. The second condition states that utilizing any number of Type I vehicles is more costly than utilizing the same number of Type II vehicles. The third condition has two implications. First, the incremental cost of using one more Type I vehicle exceeds that of an additional Type II vehicle. Secondly, the transportation cost functions are increasing in the number of vehicles used. Similarly, the fourth and the fifth conditions state that $H_{t}(I)$ and $W_{t}(w)$ are increasing functions of $I$ and $w$, respectively. In this setting, the manufacturer has to decide for each period (i) how many units to produce, (ii) how many units of each order to deliver, and (iii) how many vehicles of each type to use. Before we proceed with a mathematical model for the manufacturer to make these decisions optimally, let us define the parameters and the decision variables.

Parameters

$\mathcal{N}$ : Set of orders.

$T$ : Number of periods.

$P_{t}$ : Production capacity in period $t$.

$S_{i}$ : Size of order $i$ in number of units.

$D_{i}$ : Deadline by which to deliver all items of order $i$.

$K$ : Capacity of a vehicle in number of units.

$A_{t}$ : Number of Type II vehicles available in period $t$.

$H_{t}(I)$ : Cost of carrying $I$ units of inventory from period $t$ to $t+1$.

$C_{1, t}(x)$ : Cost of utilizing $x$ number of Type I vehicles in period $t$.

$C_{2, t}(x)$ : Cost of utilizing $x$ number of Type II vehicles in period $t$.

$W_{t}(x)$ : Cost of holding $x$ number of Type II vehicles from period $t$ to $t+1$.

\section{Decision variables}

$\pi_{t}$ : Number of items produced in period $t$.

$\pi_{t, i}$ : Number of items produced in period $t$ for order $i$.

$I_{t, i}$ : Inventory level for items of order $i$ at the end of period $t$.

$I_{t}$ : Total inventory at the end of period $t$.

$x_{t}$ : Number of Type II vehicles utilized in period $t$.

$w_{t}$ : Number of Type II vehicles carried from period $t$ to $t+1$.

$\sigma_{t, i}$ : Number of items of order $i$ delivered in period $t$.

$\tilde{\sigma}_{t, i}: \begin{cases}1, & \text { if order } i \text { is delivered in period } t \\ 0, & \text { otherwise. }\end{cases}$

$\theta_{t}$ : Total number of vehicles utilized for deliveries in period $t$.

$\theta_{t, i}$ : Number of vehicles utilized for delivery of order $i$ in period $t$.

Model 1 incorporates formulations for the six problems. Some of its constraints should be employed under all delivery characteristics (e.g., Expressions (1) through (5)). Others are applicable only in certain cases depending on whether splitting and/or consolidation are allowed. These constraints are labeled with the abbreviation we have adopted for each delivery characteristic. For example, the label in parenthesis alongside Expression (6) (i.e., $(S)$ ), indicates that this constraint should be used when orders can be split.

The objective of Model 1 is to minimize the sum of Types I and II vehicle costs, waiting costs of Type II vehicles and inventory holding costs in all periods. Constraint (1) ensures that the demand for Type II vehicles in period $t$ (those are either utilized in period $t$ or carried to period $t+1$ ) does not exceed the supply of Type II vehicles in period $t$ (those that have been recently available or been carried from period $t-1$ ). Eqs. (2) and (3) represent the total production and inventory quantities in a period in terms of those for individual orders. Constraint (4) ensures that the 
number of Type II vehicles utilized in period $t$ does not exceed the total number of vehicles used for outbound transportation in the same period. Constraint (5) sets the production capacity of period $t$ as an upper bound on the total quantity produced in period $t$. Inventory balance is maintained by either Eq. (6) or Eq. (8), depending on whether splitting orders is allowed or not. Similarly, deadlines are enforced by either Constraint (7) or Constraint (9). Vehicle capacities are modeled by one of the following constraint sets: (10)-(12) or (14). Constraints (13) and (15) establish the relation between the number of vehicles allocated for the delivery of individual orders and the total number of vehicles used in a period. Finally, Expressions (16)-(19) set nonnegativity, integrality and initial conditions on variables.

Model 1: generic formulation

Minimize $\sum_{t=1}^{T}\left[C_{1, t}\left(\theta_{t}-x_{t}\right)+C_{2, t}\left(x_{t}\right)+W_{t}\left(w_{t}\right)\right]+\sum_{t=1}^{T} H_{t}\left(I_{t}\right)$

subject to

$$
\begin{array}{ll}
x_{t}+w_{t} \leq A_{t}+w_{t-1} & t=1, \ldots, T \\
\sum_{i \in \mathcal{N}} \pi_{t, i}=\pi_{t} & t=1, \ldots, T \\
\sum_{i \in \mathcal{N}} I_{t, i}=I_{t} & t=1, \ldots, T \\
x_{t} \leq \theta_{t} & t=1, \ldots, T \\
\pi_{t} \leq P_{t} & t=1, \ldots, T
\end{array}
$$

$$
\begin{array}{lr}
I_{t, i}=I_{t-1, i}+\pi_{t, i}-\sigma_{t, i} & t=1, \ldots, T, \forall i \in \mathcal{N} \\
\sum_{t=1}^{D_{i}} \sigma_{t, i}=S_{i} & \forall i \in \mathcal{N} \\
I_{t, i}=I_{t-1, i}+\pi_{t, i}-\tilde{\sigma}_{t, i} S_{i} & t=1, \ldots, T, \forall i \in \mathcal{N} \\
\sum_{t=1}^{D_{i}} \tilde{\sigma}_{t, i}=1 & \forall i \in \mathcal{N}
\end{array}
$$

\begin{tabular}{|c|c|c|}
\hline$\tilde{\sigma}_{t, i}\left\lceil S_{i} / K\right\rceil=\theta_{t, i}$ & $t=1, \ldots, T, \forall i \in \mathcal{N}$ & $(n C-n S)$ \\
\hline$\sum \theta_{t, i}=\theta_{t}$ & $t=1, \ldots, T$ & $(n C-n S)$ \\
\hline
\end{tabular}

$\sum_{i \in \mathcal{N}} \sigma_{t, i} \leq \theta_{t} K$

$$
\begin{aligned}
t=1, \ldots, T & (C-S) \\
\text { or } & \\
t=1, \ldots, T & (C-n S)
\end{aligned}
$$

$\sum_{i \in \mathcal{N}} \tilde{\sigma}_{t, i} S_{i} \leq \theta_{t} K$

or

$\sigma_{t, i} \leq \theta_{t, i} K$

$$
t=1, \ldots, T, \forall i \in \mathcal{N} \quad(n C-S)
$$

$\sum_{i \in \mathcal{N}} \theta_{t, i}=\theta_{t}$

$t=1, \ldots, T$

$(n C-S)$

or

\section{Optimality properties}

In this section, we present some common structural properties that the optimal solutions of the six problems exhibit. In later parts of the paper, further analysis of each problem will be developed.
Theorem 1. In an optimal solution, either the inventory of Type II vehicles at the start of a period is positive or the number of Type II vehicles that are released at the end of the same period is positive, but not both. That is, $\left[A_{t}+w_{t-1}-\left(x_{t}+w_{t}\right)\right] w_{t-1}=0$ for $t=1,2, \ldots, T$.

Proof. As $w_{0}=0$, the theorem holds for $t=1$ trivially. For the other periods, the proof will follow by contradiction. That is, assume there exists an optimal solution $S$ where $\left[A_{\tau}+w_{\tau-1}-\right.$ $\left.\left(x_{\tau}+w_{\tau}\right)\right] w_{\tau-1}>0$ at some period $\tau$ (i.e., $\left.\tau \geq 2\right)$. This is possible only if $\left[A_{\tau}+w_{\tau-1}-\left(x_{\tau}+w_{\tau}\right)\right]>0$ and $w_{\tau-1}>0$. Now, consider another solution $S^{\prime}$ with everything being the same except $w_{\tau-1}^{\prime}=$ $w_{\tau-1}-1$. Clearly, $w_{\tau-1}^{\prime} \geq 0$ and $\left[A_{\tau}+w_{\tau-1}^{\prime}-\left(x_{\tau}+w_{\tau}\right)\right] \geq 0$. $S^{\prime}$ is feasible and the objective function value of $S^{\prime}$ is smaller than that of $S$ by an amount of $W_{\tau-1}\left(w_{\tau-1}\right)-W_{\tau-1}\left(w_{\tau-1}-1\right)$. This contradicts with the optimality of $S$.

Theorem 2. In an optimal solution, either the number of Type I vehicles hired in a period is positive or the number of Type II vehicles that are released at the end of the same period is positive, but not both. That is, $\left[A_{t}+w_{t-1}-\left(x_{t}+w_{t}\right)\right]\left(\theta_{t}-x_{t}\right)=0$ for $t=1,2, \ldots, T$.

Proof. Proof is by contradiction. Let $S$ be an optimal solution and $\tau$ be a period in which $\left[A_{\tau}+w_{\tau-1}-\left(x_{\tau}+w_{\tau}\right)\right]\left(\theta_{\tau}-x_{\tau}\right)>0$. Consider another solution $S^{\prime}$ with $x_{\tau}^{\prime}=x_{\tau}+1$ and everything else being the same as in $S$. Since $\left(\theta_{\tau}-x_{\tau}\right)>0$ and $A_{\tau}+w_{\tau-1}>x_{\tau}+w_{\tau}$, it turns out that $\left(\theta_{\tau}-x_{\tau}^{\prime}\right) \geq 0$ and $A_{\tau}+w_{\tau-1} \geq x_{\tau}^{\prime}+w_{\tau} . S^{\prime}$ is feasible and the objective function value of $S^{\prime}$ is smaller than that of $S$ by an amount of $C_{1, \tau}\left(\theta_{\tau}-x_{\tau}\right)+C_{2, \tau}\left(x_{\tau}\right)-C_{1, \tau}\left(\theta_{\tau}-x_{\tau}-1\right)-C_{2, \tau}\left(x_{\tau}+1\right)>$ 0 . This contradicts with the optimality of $S$.

Theorem 3. In an optimal solution, either the inventory of items at the start of a period is positive or the facility does not produce at full capacity in the same period, but not both. That is, $\left(P_{t}-\pi_{t}\right) I_{t-1}=0$ for $t=1,2, \ldots, T$.

Proof. As $I_{0}=0$, the theorem holds for $t=1$ trivially. For the other periods, the proof will follow by contradiction. Assume that there exists an optimal solution $S$ with a period $\tau$ (i.e., $\tau \geq 2$ ) having $\left(P_{\tau}-\pi_{\tau}\right) I_{\tau-1} \neq 0$. This implies $P_{\tau}>\pi_{\tau}$ and $I_{\tau-1}>0$. Therefore, there is an order $i$ for which the total quantity produced within the first $\tau-1$ periods exceeds the total amount delivered. That is,

$I_{\tau-1, i}=\sum_{k=1}^{\tau-1} \pi_{k, i}-\sum_{k=1}^{\tau-1} \sigma_{k, i}>0$.

Let $v$ be the latest production period before $\tau$ for order $i$. That is, $v=\max \left\{k: \pi_{k, i}>0, k<\tau\right\}$. We know that such $v$ exists as $\sum_{k=1}^{\tau-1} \pi_{k, i}>0$. Note that $\sum_{k=v+1}^{\tau-1} \pi_{k, i}=0$, by selection of $v$. Therefore,

$\sum_{k=1}^{\tau-1} \pi_{k, i}=\sum_{k=1}^{v} \pi_{k, i}+\sum_{k=v+1}^{\tau-1} \pi_{k, i}=\sum_{k=1}^{v} \pi_{k, i}$.

Combining Expression (20) with Expression (21) leads to $\sum_{k=1}^{v} \pi_{k, i}$ $>\sum_{k=1}^{\tau-1} \sigma_{k, i}$, which further implies that $I_{t, i}>0$ and $I_{t}>0, \forall t=$ $v, v+1, \ldots, \tau-1$. Now, consider another solution $S^{\prime}$ such that

$\pi_{\tau, i}^{\prime}=\pi_{\tau, i}+1$,

$\pi_{v, i}^{\prime}=\pi_{v, i}-1$,

$I_{t, i}^{\prime}=I_{t, i}-1, \quad \forall t=v, v+1, \ldots, \tau-1$,

$I_{t}^{\prime}=I_{t}-1, \quad \forall t=v, v+1, \ldots, \tau-1$.

Observe that, in this new solution $S^{\prime}$, we have $\pi_{\tau}^{\prime} \leq P_{\tau}$ and $\sum_{k=1}^{v} \pi_{k, i}^{\prime} \geq \sum_{k=1}^{\tau-1} \sigma_{k, i}$. Therefore, $S^{\prime}$ is feasible. Furthermore, $S^{\prime}$ 
has an objective function value smaller than that of $S$ by an amount equal to $\sum_{k=v}^{\tau-1}\left[H_{k}\left(I_{k}\right)-H_{k}\left(I_{k}-1\right)\right]>0$. Therefore $S$ is not an optimal solution.

Theorem 4. If all the cost functions are linear in their arguments and are the same in all periods, then in an optimal solution, either the number of Type I vehicles hired in a period is positive or the number of Type II vehicles carried to the next period is positive, but not both. That is, if $C_{1, t}(x)=C_{1} x, C_{2, t}(x)=C_{2} x, H_{t}(x)=H x$, and $W_{t}(x)=W x$, then $\left(\theta_{t}-x_{t}\right) w_{t}=0$ for $t=1, \ldots, T$.

Proof. The proof will follow by contradiction. That is, assume there exists an optimal solution $S$ where $\left(\theta_{\tau}-x_{\tau}\right) w_{\tau} \neq 0$ at some period $\tau$ (i.e., $\tau \geq 2$ ). Then, due to Eqs. (4) and (19), we have $\theta_{\tau}-x_{\tau}>0$ and $w_{\tau}>0$. Let $v$ be the first period after $\tau$ that has its ending inventory of Type II vehicles as zero. That is, $w_{v}=0$ and $w_{t}>0$ for $\tau \leq t<v$. Theorem 1, jointly with the fact that $w_{T}=0$, implies that there exists such a period $v$ and $x_{v}>0$. Now construct another solution $S^{\prime}$ by making the following changes on $S$ :

$x_{\tau}^{\prime}=x_{\tau}+1$,

$w_{t}^{\prime}=w_{t}-1, \quad \forall t: \tau \leq t<v$,

$x_{v}^{\prime}=x_{v}-1$.

Since $x_{\tau}^{\prime}=x_{\tau}+1$ and $w_{\tau}^{\prime}=w_{\tau}-1$, we have $x_{\tau}^{\prime}+w_{\tau}^{\prime}=x_{\tau}+w_{\tau}$. Furthermore, $A_{\tau}+w_{\tau-1}^{\prime}=A_{\tau}+w_{\tau-1}$. Therefore, Constraint (1) still holds for period $\tau$ of new solution $S^{\prime}$ (i.e., $x_{\tau}^{\prime}+w_{\tau}^{\prime} \leq A_{\tau}+w_{\tau-1}^{\prime}$ ). For $t=\tau+1, \tau+2, \ldots, v-1$, we have $x_{t}^{\prime}+w_{t}^{\prime}=x_{t}+w_{t}-1$ and $A_{t}+w_{t-1}^{\prime}=A_{t}+w_{\tau-1}-1$. Therefore, $x_{t}^{\prime}+w_{t}^{\prime} \leq A_{t}+w_{t-1}^{\prime}$, and hence, Constraint (1) holds for periods $t=\tau+1, \tau+2, \ldots, v-1$ of $S^{\prime}$ as well. As $x_{\tau}<\theta_{\tau}$ and $x_{v}>0$, it follows that $x_{\tau}^{\prime} \leq \theta_{\tau}$ and $x_{v}^{\prime} \geq$ 0 , respectively. Therefore, $S^{\prime}$ is a feasible solution. Furthermore, the objective function value of $S^{\prime}$ is smaller than that of $S$ by an amount of $(\tau-t) W>0$. Therefore, $S$ is not an optimal solution.

\section{Problems with General-Delivery characteristic}

In this section, we further analyze the four problems in which order sizes are not required to be integer multiples of the vehicle capacity. We start with the case where both consolidation and splitting are allowed.

\subsection{Problem 1: consolidate and split policy}

In this setting, the manufacturer can consolidate multiple orders and deliver them in the same vehicle. Moreover, orders can be split and delivered in different periods. Using the fivefield notation in Chen [1], this problem can be represented as $1\left|\bar{d}_{j}\right| V_{1}(\infty, Q), V_{2}\left(v_{t}, Q\right)$, split $|n|(T C+I H C)$. The two entries in the third field of the representation scheme identify the characteristics of the two vehicle types. The notation $v_{t}$ signifies that the second vehicle type has finite and time-varying availability. TC and $Q$, as defined in Chen [1], stand for transportation costs and size of capacitated vehicles. Note that the value of $Q$ in our paper is $K$, and we use $I H C$ as an abbreviation for inventory holding costs.

The following theorem implies that the production and delivery sequences in Problem 1 can be optimally determined. Even though this significantly alleviates the difficulties of the original problem, the problem of finding the production and the delivery quantities still needs to be solved.

Theorem 5. There is an optimal solution to Problem 1, in which orders are produced and delivered in nondecreasing order of delivery deadlines.
Proof. The proof will follow by showing that, given an optimal solution, an alternative one in which orders are produced and delivered in nondecreasing order of delivery deadlines can be obtained. This will be achieved by keeping the total production and delivery quantities in each period the same, but changing the allocation of items produced to different orders.

Now, consider an optimal solution. Define $\sigma_{t}$ as the total quantity delivered in period $t$ of this solution. Also, let $T P(t)$ and $T S(t)$ be the total quantities produced by period $t$ and delivered by period $t$, respectively. That is,

$\sigma_{t}=\sum_{i \in \mathcal{N}} \sigma_{t, i}, \quad T P(t)=\sum_{k=1}^{t} \pi_{k}, \quad T S(t)=\sum_{k=1}^{t} \sigma_{k}$.

Without loss of generality assume that $D_{1} \leq D_{2} \leq \cdots \leq D_{|\mathcal{N}|}$, and let the total size of the first $i$ orders in this sequence be denoted by $T D(i)$. That is, $T D(i)=\sum_{j=1}^{i} S_{j}$.

Consider another solution where the first $S_{1}$ units produced and delivered are assigned to order 1 , the next $S_{2}$ units are assigned to order 2 , and so on. It is important to note that the consolidate-split policy enables this kind of a reassignment. More specifically, the amount of production for order $i$ in period $t$ of this new solution is as follows:

$$
\begin{aligned}
\pi_{t, i}^{\prime}= & \min \left\{S_{i}, \pi_{t}, \max \{T D(i)-T P(t-1), 0\},\right. \\
& \max \{T P(t)-T D(i-1), 0\}\} .
\end{aligned}
$$

The expression for $\pi_{t, i}^{\prime}$ states that the production amount for order $i$ in period $t$ is now the minimum of the following: size of order $i$; production amount in period $t$; of all the production in period $t$, the amount dedicated for order $i$ if the production in the first $t-1$ periods satisfies a partial amount of order $i$ after meeting the requirements of the first $i-1$ orders; the remaining amount of period $t$ 's production that is dedicated for order $i$ after satisfying the demand for the first $i-1$ orders. An assignment of delivery quantities over periods to different orders can similarly be done using the following expression:

$$
\begin{aligned}
\sigma_{t, i}^{\prime}= & \min \left\{S_{i}, \sigma_{t}, \max \{T D(i)-T S(t-1), 0\},\right. \\
& \max \{T S(t)-T D(i-1), 0\}\} .
\end{aligned}
$$

Since total production and delivery sizes remain the same, the cost of the new solution is the same as that of the original solution. This proves that the new solution is also optimal.

Using Theorem 5, the generic multi-order model discussed in Section 2 can be rewritten as if there is a single order. The solution to this simplified model should then be converted to a solution for the original problem by assigning the first $S_{1}$ units to order 1 , the next $S_{2}$ units to order 2, and so forth. Before we proceed with this model, let us define $\delta_{t}$ as the total size of orders having period $t$ as their deadlines. That is,

$\delta_{t}=\sum_{i: D_{i}=t} S_{i} \quad \forall t=1, \ldots, T$.

Model 2: single-order formulation for Problem 1

Minimize $\sum_{t=1}^{T}\left\{C_{1, t}\left(\theta_{t}-x_{t}\right)+C_{2, t}\left(x_{t}\right)+W_{t}\left(w_{t}\right)\right\}+\sum_{t=1}^{T} H_{t}\left(I_{t}\right)$

subject to

$x_{t}+w_{t} \leq A_{t}+w_{t-1} \quad t=1, \ldots, T$

$x_{t} \leq \theta_{t} \quad t=1, \ldots, T$

$\pi_{t} \leq P_{t} \quad t=1, \ldots, T$

$I_{t}=I_{t-1}+\pi_{t}-\sigma_{t} \quad t=1, \ldots, T$

$\sum_{k=1}^{t} \sigma_{k} \geq \sum_{k=1}^{t} \delta_{k} \quad t=1, \ldots, T$ 
$\theta_{t} K \geq \sigma_{t} \quad t=1, \ldots, T$

$w_{0}=I_{0}=0$

$I_{t}, \sigma_{t}, \pi_{t}, w_{t}, x_{t}, \theta_{t} \in \mathcal{Z}^{+} \cup\{0\} \quad t=1, \ldots, T$.

Below, we propose a dynamic programming formulation which solves this problem in pseudo-polynomial time. Existence of such an algorithm shows that the manufacturer's planning problem under the Consolidate and Split policy may be $\mathcal{N} \mathcal{P}$-hard but not $\mathcal{N} \mathcal{P}$-hard in the strong sense.

Algorithm 1. Define $C(t, \pi, \sigma, w)$ as the minimum total cost accumulated at the end of period $t$, when the total production and delivery quantities in the first $t$ periods are $\pi$ and $\sigma$, respectively, and the number of vehicles held to the next period at the end of period $t$ is $w$.

Initial conditions:

$C(0,0,0,0)=0$

$C(t, \pi, \sigma, w)=\infty \quad \forall t, \pi, \sigma, w: \min (t, \pi, \sigma, w)<0$.

Recursive relation:

$C(t, \pi, \sigma, w)$

$$
= \begin{cases}\infty, & \text { if } \pi<\sigma, \\ \infty, & \text { if } \sigma<\sum_{i: D_{i} \leq t} S_{i}, \\ \min _{\substack{x(t, \pi, \sigma, w) \\ x_{t}+w_{t} \leq A_{t}+w_{t-1}}}\left\{C\left(t-1, \pi-\pi_{t}, \sigma-\sigma_{t}, w_{t-1}\right)\right. \\ \left.+C_{1, t}\left(\theta_{t}-x_{t}\right)+C_{2, t}\left(x_{t}\right)+H_{t}(\pi-\sigma)+W_{t}\left(w_{t}\right)\right\}, \\ \text { o.w., }\end{cases}
$$

where

$$
\begin{aligned}
\mathcal{X}(t, \pi, \sigma, w)=\{ & \left(\pi_{t}, \sigma_{t}, x_{t}, \theta_{t}, w_{t}\right) \mid \pi_{t} \leq P_{t}, \\
& \left.w_{t} \leq w, \sigma_{t} \leq K \theta_{t}, x_{t} \leq \theta_{t}\right\} .
\end{aligned}
$$

Optimal solution value: $C(T, \hat{D}, \hat{D}, 0)$, where $\hat{D}=\sum_{i \in \mathcal{N}} S_{i}$.

The computational complexity of Algorithm 1 is presented in the next lemma and proved in the online appendix.

Lemma 1. Algorithm 1 finds an optimal solution for Problem 1 in $O\left(T \hat{D}^{6} W^{2} / K^{2}\right)$ time, where $W=\min \left(\hat{D} / K, \sum_{i=1}^{T} A_{i}\right)$.

\subsection{Problem 2: no-consolidate and split policy}

In this problem, different orders cannot be consolidated but an order can be delivered in partial shipments over time. Using Chen [1]'s five-field notation, this problem can be represented as $1\left|\bar{d}_{j}\right| V_{1}(\infty, Q), V_{2}\left(v_{t}, Q\right)$, direct, split $|n|(T C+I H C)$. The following theorem and its proof imply that Problem 2 is $\mathcal{N} \mathcal{P}$-hard in the strong sense even for the linear cost structure.

Theorem 6. Problem 2 is $\mathcal{N} \mathcal{P}$-hard in the strong sense.

Proof. Proof is done by a reduction from the 3-Partition (3P) problem. Note that Problem 2 is clearly in $\mathcal{N} \mathcal{P}$. 3P is defined as follows:

INSTANCE: Set $g$ of $3 m$ elements, a bound $B \in Z^{+}$, and a size $s(a) \in Z^{+}$for each $a \in g$ such that $B / 4<s(a)<B / 2$ and such that $\sum_{a \in g} s(a)=m B$.

QUESTION: Can $g$ be partitioned into $m$ disjoint sets $g_{1}, g_{2}, \ldots$, $g_{m}$ such that $\sum_{a \in g_{\tau}} s(a)=B$ for $\tau=1,2, \ldots, m$ (note that each $g_{\tau}$ must therefore contain exactly three elements from $\left.g\right)$ ?

REDUCTION: Take an arbitrary instance of 3P. The corresponding instance of Problem 2 is constructed as follows: set $\mathcal{N}=g$,

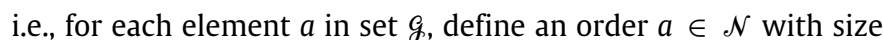
$S_{a}=s(a)$. Furthermore, set $T=m, K=B, P_{t}=B$ for all $t=1, \ldots, T, D_{a}=T$ for all $a$ in $\mathcal{N}$, and $A_{t}=3, C_{1, t}(x)=2 x$, $C_{2, t}(x)=H_{t}(x)=W_{t}(x)=x$ for $t=1,2, \ldots, T$. We will show that there is a solution to $3 \mathrm{P}$ if and only if there is a solution to Problem 2 with cost less than or equal to $z^{*}=3 m$.

Assume that there is a solution to Problem 2 with $\operatorname{cost} z$, which is less than or equal to $z^{*}=3 m$. Since there are $3 m$ orders and they cannot be consolidated, the cost of transporting these orders is at least $3 m$. This implies the total cost is exactly $3 m$, which, in turn, is possible only if all Type II vehicles are utilized, and no inventory or vehicle holding cost is incurred. As a result, exactly three orders are completed and delivered in each period. Moreover, the total number of items produced in each period is equal to $B$. Now construct a solution to 3P as follows: for all orders produced and delivered in period $t$, put the corresponding element of set $g$ into $g_{t}$. As the size of orders $S_{a}=s(a)$, for each disjoint set $g_{t}$, $\sum_{a \in g_{t}} s(a)=B(t=1,2, \ldots, m)$.

If there is a solution to $3 \mathrm{P}$, a solution to Problem 2 can be constructed as follows: for each disjoint set $g_{t}, \tau=1,2, \ldots, m$, produce and deliver all the items of order $a \in g_{t}$ in period $t$. A similar reduction as in the previous case implies that the solution has a cost of $z=3 m \leq z^{*}$.

\subsection{Problem 3: consolidate and no-split policy}

In this problem, orders can be consolidated, however, an order cannot be delivered in partial shipments over time. According to Chen [1]'s representation scheme, this problem corresponds to $1\left|\bar{d}_{j}\right| V_{1}(\infty, Q), V_{2}\left(v_{t}, Q\right)|n|(T C+I H C)$. In the next theorem, we establish its complexity status.

Theorem 7. Problem 3 is $\mathcal{N} \mathcal{P}$-hard in the strong sense.

Proof. Similar to the proof of Theorem 6 with $A_{t}=1$ for each $t=1,2, \ldots, T$ and $z^{*}=m$.

\subsection{Problem 4: no-consolidate and no-split policy}

In this problem, neither consolidation nor splitting is allowed. Based on Chen [1]'s representation scheme, this problem corresponds to $1\left|\bar{d}_{j}\right| V_{1}(\infty, Q), V_{2}\left(v_{t}, Q\right)$, direct $|n|(T C+I H C)$. As stated in the following theorem, the problem is $\mathcal{N} \mathcal{P}$-hard in the strong sense even for the linear cost structure.

Theorem 8. Problem 4 is $\mathcal{N} \mathcal{P}$-hard in the strong sense.

Proof. Similar to the proof of Theorem 6 with $A_{t}=3$ for each $t=1,2, \ldots, T$ and $z^{*}=3 m$.

\section{Problems with FTL-Delivery characteristic}

For the problems discussed in this section, vehicles are required to be fully utilized in outbound transportation and therefore the size of orders must be integer multiples of vehicle capacity. In other words, the number of items in each vehicle is either 0 or $K$. We first begin with presenting two theorems that are valid for both Problems 5 and 6.

Theorem 9. If the production capacity in each period is an integer multiple of the vehicle capacity, then the production quantity in each period of an optimal solution is an integer multiple of the vehicle capacity. That is, if $\exists n_{t} \in \mathcal{Z}^{+} \cup\{0\}$ such that $P_{t}=n_{t} K$, for $t=1,2, \ldots, T$, then $\exists m_{t} \in \mathcal{Z}^{+} \cup\{0\}$ such that $\pi_{t}=m_{t} K$ for $t=1,2, \ldots, T$. 
Proof. Proof is by contradiction. Assume that there exists an optimal solution $S$ with some periods in which the production quantity is not an integer multiple of the vehicle capacity. Let $t$ be the latest such period. This implies that $\pi_{t}<P_{t}$ and $\frac{\sum_{k=t+1}^{T} \pi_{k}}{K}$ is an integer. Since all orders are integer multiples of the vehicle capacity, it follows that $\frac{\sum_{k=1}^{T} \pi_{k}}{K}$ is also an integer. The integrality of both $\frac{\sum_{k=t+1}^{T} \pi_{k}}{K}$ and $\frac{\sum_{k=1}^{T} \pi_{k}}{K}$ further implies the integrality of $\frac{\sum_{k=1}^{t} \pi_{k}}{K}$. As $\frac{\pi_{t}}{K}$ is not an integer, $\frac{\sum_{k=1}^{t-1} \pi_{k}}{K}$ is neither. Note also that, due the characteristic of the delivery policy, $\frac{\sum_{k=1}^{t-1} \sigma_{k}}{K}$ is an integer. Combining the last two results (i.e., $\frac{\sum_{k=1}^{t-1} \sigma_{k}}{K}$ is integer but $\frac{\sum_{k=1}^{t-1} \pi_{k}}{K}$ is not), we have $\sum_{k=1}^{t-1} \pi_{k}>\sum_{k=1}^{t-1} \sigma_{k}$. This implies there is at least $\left\lceil\frac{\pi_{t}}{K}\right\rceil K-\pi_{t}$ units of inventory carried from period $t-1$ to period $t$. Let $i$ be the index of the order with the largest amount of inventory at the end of period $t-1$ (i.e., $i=\operatorname{argmax}_{j}\left\{I_{t-1, j}\right\}$ ), and let $\tau$ be the latest period before $t$ in which there is some production for order $i$ (i.e., $\tau=\operatorname{argmax}_{k<t}\left\{\pi_{k, i}>0\right\}$ ).

Now, consider another solution $S^{\prime}$ with the following modification on solution $S$ :

$$
\begin{aligned}
& \pi_{\tau, i}^{\prime}=\pi_{\tau, i}-1 \\
& \pi_{t, i}^{\prime}=\pi_{t, i}+1 \\
& I_{t^{\prime}, i}^{\prime}=I_{t^{\prime}, i}-1, \quad \text { for } t^{\prime}=\tau, \tau+1, \ldots, t-1 \\
& I_{t^{\prime}}^{\prime}=I_{t^{\prime}}-1, \quad \text { for } t^{\prime}=\tau, \tau+1, \ldots, t-1 .
\end{aligned}
$$

The new solution $S^{\prime}$ has a lower objective function value than that of $S$ by an amount $\sum_{k=\tau}^{t-1} H_{k}\left(I_{k}\right)-\sum_{k=\tau}^{t-1} H_{k}\left(I_{k}-1\right)$. As $H_{t}(x)$ is an increasing function of $x$ for $t=1, \ldots, T$, it follows that the cost difference is positive. Hence, $S$ is not an optimal solution.

Theorem 10. If the production capacity in each period is an integer multiple of the vehicle capacity, then there exists an optimal solution in which the production quantity for each order is an integer multiple of the vehicle capacity in every period. That is, if $\exists n_{t} \in Z^{+} \cup$ $\{0\}$ such that $P_{t}=n_{t} K$, for $t=1,2, \ldots, T$, then there is an optimal solution in which $\exists m_{t, i} \in \mathcal{Z}^{+} \cup\{0\}$ such that $\pi_{t, i}=m_{t, i} K \forall i \in N$ for $t=1,2, \ldots, T$.

Proof. Proof is by construction. Consider an optimal solution $S$ in which some orders have production quantity which is not an integer multiple of the vehicle capacity. Note that the total production at each period is an integer multiple of the vehicle capacity due to Theorem 9 . Let $i$ be the smallest indexed order with this property and let $t$ and $\tau(t<\tau)$ be the last two periods where production of order $i$ is not an integer multiple of vehicle capacity (i.e., $\frac{\pi_{\tau, i}}{K}$ and $\frac{\pi_{t, i}}{K}$ are not integer). Note that $\frac{\sum_{k=1}^{t} \pi_{k, i}}{K}>$ $\left\lfloor\frac{\sum_{k=1}^{t} \pi_{k, i}}{K}\right\rfloor \geq \frac{\sum_{k=1}^{t} \sigma_{k, i}}{K}$. This means that a portion of production quantity for order $i$ at period $t$ can be moved to period $\tau$. As total production quantity for all periods is an integer multiple of vehicle capacity, $\exists j \in N: \pi_{\tau, j}-\left\lfloor\frac{\pi_{\tau, j}}{K}\right\rfloor K>0$. Also note that $j>i$ (as $i$ is the smallest indexed order with production not being an integer multiple of vehicle capacity). Let

$\Delta=\min \left\{\left(\pi_{\tau, j}-\left\lfloor\frac{\pi_{\tau, j}}{K}\right\rfloor K\right),\left(\left\lceil\frac{\pi_{\tau, i}}{K}\right\rceil K-\pi_{\tau, i}\right)\right\}$

and set

$\pi_{\tau, i} \leftarrow \pi_{\tau, i}+\Delta$
$\pi_{\tau, j} \leftarrow \pi_{\tau, j}-\Delta$
$\pi_{t, i} \leftarrow \pi_{t, i}-\Delta$
$\pi_{t, j} \leftarrow \pi_{t, j}+\Delta$.

Repeat the same argument until $\frac{\pi_{\tau, i}}{K}$ is integer. Note that it takes at most $|N|-j$ steps. Then, select different $t$ and $\tau$ and repeat the same arguments until $\frac{\pi_{t, i}}{K}$ is integer for all $t=1,2, \ldots, T$. Note that during this process, no orders with index less than $i$ is altered. Repeating the same procedure for all $i \in N$ results in a solution where the production quantity for each order is an integer multiple of the vehicle capacity in each period.

\subsection{Problem 5: FTL-delivery with split}

Observe that the structure of this problem is similar to that of Problem 1 . Therefore, Theorem 5 is also valid for this problem. With the same reasoning, Model 2 can be used after some modifications. Specifically, as $\sigma_{t}=K \theta_{t}$, we plug in $K \theta_{t}$ in place of $\sigma_{t}$ and update some of the decision variables and parameters as follows: $\pi_{t}=$ $K \pi_{t}^{K}, P_{t}=K P_{t}^{K}, I_{t}=K I_{t}^{K}, \delta_{t}=K \delta_{t}^{K}$ for $t=1, \ldots, T$. In this new model, the decision variables are $I_{t}^{K}, \pi_{t}^{K}, w_{t}, x_{t}$, and $\theta_{t}$, all of which are nonnegative integers.

A modified version of Algorithm 1 can be used to solve this problem in $O\left(T \hat{D}^{5} W^{2} / K^{2}\right)$ time, where $W=\min \left(\hat{D} / K, \sum_{i=1}^{T} A_{i}\right)$ and $\hat{D}$ is the cumulative demand. Note that the time complexity when Algorithm 1 is applied to Problem 5 is less, because $\sigma_{t} \leq K \theta_{t}$ is replaced by $\sigma_{t}=K \theta_{t}$.

\subsection{Problem 6: FTL-delivery with no-split}

Theorem 11. Problem 6 is $\mathcal{N} \mathcal{P}$-hard in the strong sense.

Proof. Similar to that of Theorem 6 with $A_{t}=P_{t}=B$ for each $t=1,2, \ldots, T, K=1$ and $z^{*}=B T$.

\section{Final remarks}

In order to gain more insights into the average-case complexities of the pseudo-polynomial-time algorithms in this paper, we performed a small-scale computational experiment using Algorithm 1. We incorporated the optimality properties of Section 3, which reduced the size of the input significantly. We observed that the average running time of Algorithm 1 changes by a lesser amount with respect to increasing values of the parameters than the worst-case complexity implies. The details of this analysis can be found in the online appendix of this paper.

It is important to note that the case of heterogeneous vehicles has been quite extensively covered within the context of joint ordering and transportation planning decisions (e.g., Sethi et al. [5]). However, consideration of heterogeneous vehicle types is a relatively new issue in the area of integrated production and outbound transportation planning, and this paper is the first to consider a setting where vehicles are different in their time-varying availability and costs. Our objective in this paper has been to present a generic model that encompasses several different delivery policies and to provide a primary analysis rather than to develop solution methods. This approach enabled us to observe that although there are some optimality properties that are common for all six problems, the type of delivery policy is a major factor that affects a problem's complexity. Based on the results of this paper, future research may consider the development of heuristics for individual problems and testing their performances.

\section{Appendix. Supplementary data}

Supplementary material related to this article can be found online at http://dx.doi.org/10.1016/j.orl.2012.11.002. 


\section{References}

[1] Z.-L. Chen, Integrated production and outbound distribution scheduling: review and extensions, Oper. Res. 58 (2010) 130-148.

[2] B. Chen, C.-Y. Lee, Logistics scheduling with batching and transportation, Eur. J. Oper. Res. 189 (2008) 871-876.

[3] Z.-L. Chen, G. Pundoor, Order assignment and scheduling in a supply chain, Oper. Res. 54 (2006) 555-572.

[4] C.-L. Li, J. Ou, Machine scheduling with pickup and delivery, Nav. Res. Logist. 52 (2005) 616-630.
[5] S.P. Sethi, H. Yan, H. Zhang, Peeling layers of an onion: inventory model with multiple delivery modes and forecast updates, J. Optim. Theory Appl. 108 (2001) 253-281.

6] K.E. Stecke, X. Zhao, Production and transportation integration for a maketo-order manufacturing company with a commit-to-delivery business mode Manuf. Serv. Oper. Manage. 9 (2007) 206-224.

[7] H. Wang, C.-Y. Lee, Production and transport logistics scheduling with two transport mode choices, Nav. Res. Logist. 52 (2005) 796-809.

[8] W. Zhong, Z.-L. Chen, M. Chen, Integrated production and distribution scheduling with committed delivery dates, Oper. Res. Lett. 38 (2010) 133-138. 